\title{
Le travail de lecture des groupes de lecteurs des archives belges du patrimoine autobiographique
}

Francine Meurice $^{1}$

\section{$\underline{\text { Abstract (En/Fr) }}$}

The reading work, done by groups of readers of APA-Bel during the writing of reviews of texts in the autobiography archives, brings together several strategies of interpretation. It would be interesting to establish the theoretical reading models that are able to describe the strategies most often requested so as to be able to determine competences of the reader who is registered in the communication tool of the APA as coenunciator of these private writings. This coenunciator profiles himself next to the enunciator of private writings, sometimes as guide to the construction of the ethos, sometimes as an agent bringing together a number of different reading strategies sometimes as witness of the writing practice following the example of a reader postulated by the original review, sometimes as an intimate reader. A number of discursive approaches from the APA fund will be proposed to read this dialogue between these different roles.

Le travail de lecture, fourni par les groupes de lecteurs de l'APA-Bel lors de la rédaction des échos de lecture des textes déposés dans les archives autobiographiques, convoque plusieurs stratégies d'interprétation. Il serait intéressant de repérer quels sont les modèles de lecture théoriques capables de décrire les stratégies les plus souvent sollicitées et ce, afin de cerner les compétences de ce lecteur qui est inscrit dans le dispositif communicationnel de l'APA comme coénonciateur de ces écrits privés. Ce coénonciateur se profile aux côtés de l'énonciateur des écrits privés tantôt comme le guide de la construction de l'éthos, tantôt comme un actant rassemblant plusieurs forces lectrices, tantôt comme un témoin de la pratique scripturale à l'instar du lecteur postulé par la critique génétique, tantôt comme un lecteur intime. Quelques configurations discursives du fonds de l'APA seront proposées pour lire ce dialogue entre ces différentes figures.

\section{Introduction}

Ecrire en «je», sur soi, à propos de soi, en nouant un pacte de transparence, celui de la confidence, est une entreprise très risquée non seulement parce qu'elle peut compromettre l'image de soi au regard des autres et de soi-même mais aussi parce que l'écriture sur ce mode

\footnotetext{
${ }^{1}$ Haute Ecole de Bruxelles, membre Apa-Bel.
} 
est une entreprise délicate, laborieuse, exigeante, traumatisante de construction et de déconstruction de la personnalité.

Quand il s'agit de littérature fictionnelle, même si le trauma est identique pour l'auteur, c'est son métier et l'institution du littéraire, même fragile, est un rempart. La mise en scène sociale de la transaction autour du livre met la distance entre ses lecteurs et lui. La fictionnalisation du vécu protège son moi d'écrivain de ce qui sans pare excitation serait trop douloureusement ressenti. C'est pourquoi, les livres et les manuscrits qui sont reçus à l'APA sont des objets délicats. Ils se défont des normes coutumières de la lecture, ils transgressent les rapports institués dans le vieux couple littéraire. L'auteur n'y fait pas semblant d'ignorer son lecteur, il ne feint pas de n'avoir nulle conscience de sa présence physique. Le lecteur se manifeste concrètement au point d'écrire lui-même en écho à sa lecture et de transmettre sa confidence de lecteur à l'auteur, par courrier, pour en espérer une réponse. Les risques ne sont pas négligeables dès lors que les deux rôles désincarnés de l'auteur et du lecteur du pacte littéraire reprennent corporéité.

La procédure de lecture de l'APA-Bel est tributaire de ce dispositif communicationnel qui réinstalle les doubles en chair et en os aux côtés du narrateur et du lecteur modèle inscrits dans le texte. Même si l'auteur n'est plus là pour déposer son manuscrit dans le patrimoine autobiographique, le déposant qui s'en charge reprend en quelque sorte la relève et devient comme une espèce de méta-auteur tutélaire.

Le lecteur du groupe de lecture s'engage dans ce processus de lecture avec des précautions et des ruses de Sioux. C'est qu'il devra rendre des comptes à cet auteur particulier et pas à lui seul, à l'APA tout entière, cette instance lectrice cautionnante. Sa tâche et sa première compétence lectrice ressemblera au travail de l'entomologiste : déplier sans déchirer, sans déformer et sans froisser le texte qui se donne à lui pour respecter le pacte de celui qui s'expose au risque de l'écrit autobiographique. Son fil conducteur est l'attention du confident sensible au non-dit autant qu'au dit comme lieux féconds en significations. Sa deuxième compétence est de mesurer les variations entre le dicible et l'indicible, non pour juger ou dévoiler sans pudeur, mais pour interpréter dans ces variations le patrimoine autobiographique dont il est tributaire. Sa méthodologie de lecture lui confère plusieurs autres compétences que je décrirai de manière plus générale après avoir montré comment l'interprétation peut se construire en partant d'un dépôt particulier. 


\section{Présentation du dépôt et de la méthodologie}

\subsection{La méthodologie}

Le dépôt que j'ai choisi d'analyser est celui de José Dosogne. Il contient six manuscrits. En montrant comment le travail de lecture peut se construire à partir de ce dépôt, mon objectif sera triple:

1. repérer les modèles de lecture convoqués qui sont sous-jacents au travail d'interprétation;

2. montrer comment l'écriture se déplace et se forge dans les variations du dit et du non-dit lors de la cristallisation des biographèmes;

3. montrer que par la pratique de l'écriture des échos de lecture, l'APA construit une instance lectorielle, une communauté discursive.

A partir de ces trois objectifs, je défendrai trois thèses:

1. les modèles de lecture convoqués correspondent aux différentes représentations de la lecture littéraire qui ont successivement fait autorité dans l'enseignement de la littérature dans les écoles ${ }^{2}$. On les retrouve dans les échos rédigés ou dans le travail d'interprétation que je vais analyser. Il n'y a donc pas de doctrine ou un format de l'écho de lecture type mais une négociation d'un consensus dans un groupe de lecteurs, puis au sein de l'APA tout entière qui se constitue en communauté discursive;

2. lire tous les textes d'un déposant ainsi que les textes associés par les thèmes ou par les liens sociaux et qui appartiennent au fonds, permet de textualiser l'ensemble du dépôt et de mieux interpréter le non-dit;

3. accentuer l'importance de la coopération du lecteur lors de la lecture et donc tenir compte de la réception des textes, correspond aux missions d'éducation populaire qui sous-tendent des associations comme l'APA. "Accueillir sans exclusive ni sélection des textes autobiographiques, reconnaître que tout individu, même le moins lettré et cultivé au sens de la culture savante est porteur d'une culture, d'un savoir, d'une richesse d'expérience, qui ont leur valeur, leur légitimité et méritent d'être explicités et reconnus comme tels» ${ }^{3}$.

\footnotetext{
2 D'après les quatre définitions de la lecture littéraire formulées par Jean Louis DUFAYS in Daniel DELBRASSINE (2007), Découvrir 'la lecture littéraire' avec les romans écrits pour la jeunesse, Namur, Presses universitaires de Namur, Tactiques $\mathrm{n}^{\circ} 2$.

${ }^{3}$ «Accueillir des textes autobiographiques, sans exclusive ni sélection a priori; leur ouvrir un droit à être lus et à faire l'objet d'un écho. On rejoint là les idéaux de l'Education Populaire. [...] La seconde signification de
} 


\subsection{Le catalogue du dépôt}

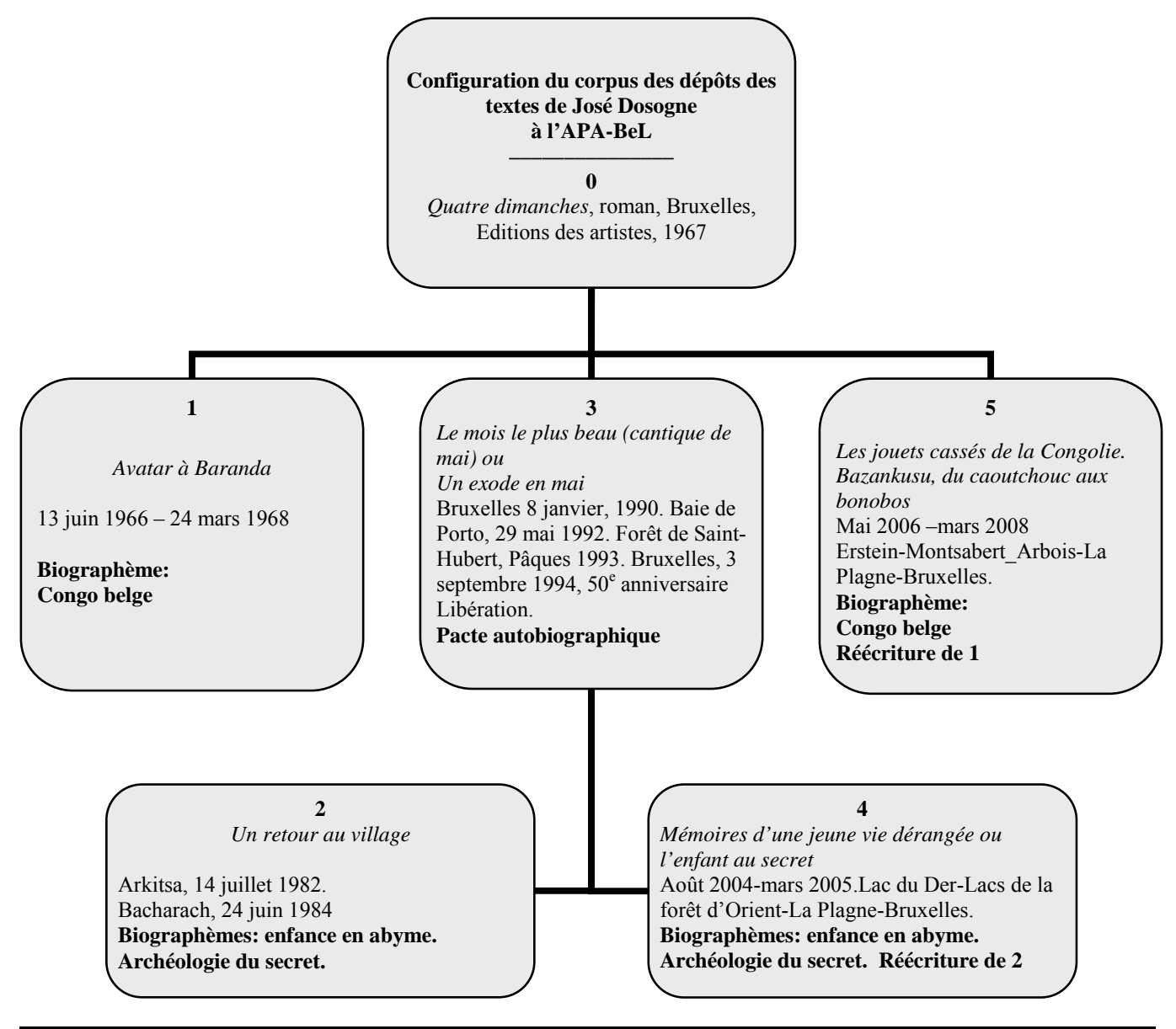

l'Education Populaire se développe principalement en France à partir de mai 68 et en liaison avec les travaux de recherche en ethnologie. Elle tient que tout individu, même le moins lettré et cultivé au sens de la culture savante, est porteur d'une culture, d'un savoir, d'une richesse d'expérience, qui ont leur valeur, leur légitimité et méritent d'être explicités et reconnus comme tels. On est là très proches de l'idée de culture populaire, voire de culture de classe». Alex LAINE, «Echos d'un observateur impliqué», in Les cahiers de l'APA, Groupes de lecture APA, Lecteurs de vie, $\mathrm{n}^{\circ} 20$, Juin 2001, p.47. 
Quatre dimanches est un roman polyphonique dans lequel une constellation de personnages vit en parallèle quatre dimanches. Le lecteur perçoit qu'il s'agit d'un éclatement de l'autobiographie, comme c'est souvent le cas dans le traitement romanesque, le moi du narrateur se réverbère dans les différents personnages qui incarnent les thèses, les motifs récurrents des textes qui suivront: le conflit parents/enfants dans la configuration spécifique d'un non-dit pernicieux de l'histoire familiale, la conquête de l'autonomie existentielle aux prises avec ses différents étouffoirs: religion, famille, convenances, amour matrimonial. Le contexte de la topographie urbaine et de l'architecture de Bruxelles ainsi que l'atmosphère des années 60 sont contemporains du moment de l'écriture et du vécu des personnages.

Le cadre d'Avatar à Baranda est le Congo belge à la veille de l'indépendance en1960, de 1953 à 1956. Ce récit polyphonique relate les monologues intérieurs des trois protagonistes: Bertrand, agent territorial, Laure, son épouse et Faustin, le boy. «Dans le temps restreint d'un week-end, comme sur une scène de théâtre, les personnages réussissent ensemble leur métamorphose» ${ }^{4}$. Bertrand prend ses distances avec le monde colonial et Laure son épouse redécouvre sa tendresse et son désir pour Bertrand. Faustin, de manière beaucoup plus voilée, œuvre à la tâche de l'indépendance. Ecrit de 1966 à 1968, ce roman autobiographique, sans pacte autobiographique explicite, se démarque des discours de l'époque par sa posture antiraciste et sa volonté de déchiffrer le non-dit du contexte colonial.

Un retour au village superpose le temps contemporain de l'écriture et celui de l'enfance lors du retour dans son village d'enfant, Gercy, d'un enseignant quinquagénaire qui doit préparer un nouveau cours de mathématique pour l'architecture. Sans l'avoir voulu, ce retour est une fouille archéologique dans le non-dit du passé. Le leitmotiv est celui du secret qui entoure la raison de l'écartement de l'enfant élevé par ses grands parents. Mais alors que ce secret est encore informulé comment l'investiguer? Au fur et à mesure du récit, les enclaves des souvenirs s'amplifient et imposent leurs réalités, sans transition, à la narration principale, celle de Bertrand, écrite à la troisième personne. Comme dans un film ou dans les rêves, ce sont des images puissantes qui affleurent alors, comme entre autres, celles des mamans de remplacement, celles des ombres de la nuit somnambule, celle des voix confuses des adultes perçues par «le trou du chauffage» dans le plancher de la chambre.

C'est à partir de Un exode en mai, que le récit autobiographique est pris en charge par la première personne. C'est après ce récit que s'enclenche un processus de réécriture des textes qui

\footnotetext{
${ }^{4}$ J. DOSOGNE, Baranda, avant propos.
} 
précédent. C'est pour cette raison que nous l'avons placé au centre du schéma d'analyse, et que nous en approfondirons la lecture ci-après.

Mémoire d'une jeune vie dérangée reprend Un retour au village en y ajoutant l'explicite. L'explicite du secret - un père maladivement jaloux impose cette abnégation de l'écartement, à son épouse et à son enfant - et l'explicite des contextes qui sont restaurés avec minutie comme c'est le cas pour Un exode en mai.

Les jouets cassés de la Congolie, du caoutchouc aux bonobos, réécrit Baranda avec une politique d'écriture similaire: expliciter de plus en plus les non-dits -coloniaux dans ce cas pour leur arracher leur côté pernicieux. Ici aussi, les contextes sociopolitiques sont restaurés avec minutie, construisant une argumentation originale et solide dénonçant l'absence d'élaboration collective et politique de cette période de l'histoire de la Belgique.

\subsection{La textualisation d'un corpus}

Le mois le plus beau (cantique de mai) ou Un exode en mai est un récit présenté comme un journal contenant des entrées quotidiennes datées du vendredi 10 mai au jeudi 23 mai. C'est le road movie du départ en exode vers la France à pied puis en train, du village de Gercy jusque Creuziez-le vieux dans le Bourbonnais. Une suite plus courte relate la période du 15 septembre 1940 au 8 mai 1945.Ces deux parties sont enchâssées comme un flash back dans une troisième datée du 10 mai 1990, l'occasion de reparler 50 ans plus tard de 1940 et de retourner au village.

Le récit autobiographique écrit en je, bien qu'écrit a posteriori, respecte le regard et le point de vue de l'enfant de 9 ans. Le narrateur postérieur, celui de 1990 ajoute, sans se montrer comme instance énonçante, les contextes et les commentaires des événements de cet exode de mai. Souvent, l'enfant est placé en observateur de ce qui est narré, bien ancré dans l'image, comme lorsqu'au cinéma, le film veut communiquer au spectateur le point de vue du personnage. Ce procédé d'écriture permet de préserver le point de vue de l'enfant de l'époque en ne lui attribuant pas les réflexions qui commentent la scène. Le point de vue de l'enfant reste dans l'implicite. Le rythme de la marche de la colonne des réfugiés scande la narration émouvante et soutenue de ce roman d'apprentissage enclavé dans la guerre: l'exode est pour Tilou l'occasion de vivre l'amitié complice avec Jockey (Justin) et la découverte de l'amour de Céline, la mort de son ami, celle de son grand-père et de ses parents. La dernière partie intitulée «le 10 mai 1990» montre deux acteurs de l'exode, Tilou et Céline, réunis au village de Gercy pour se souvenir. C'est dans cette partie que la commande d'écriture autobiographique est explicite lorsque le narrateur dit à Céline et pour lui-même: «Je sais maintenant que j'écrirai mes souvenirs» 
(p.156). C'est un futur, une proclamation d'intention pour le je du texte mais un passé pour le lecteur qui vient de les lire. Ce montage temporel habile, s'il referme le récit comme une boucle sur lui-même, laisse en même temps planer l'attente du lecteur recevant cette promesse comme encore inaccomplie au moment où elle lui est faite. La légitimation de l'autobiographie est explicite également, les personnages sont des acteurs de l'exode. L'exode est vécu comme un événement qui fait sortir l'individu hors de ses repères usuels, cette rupture du temps cyclique de la vie ordinaire justifie sa mise en discours.

L'équation articulant la légitimation du véridique et le pacte rédactionnel autobiographique noue le contrat de lecture sur le régime de croyance propre au genre autobiographie. Si la lecture s'arrête aux frontières de ce volume du corpus des textes de José Dosogne, l'interprétation ne remettra pas en doute le régime de croyance.

Or, la confrontation du scénario familial d'Un exode en mai avec celui des autres textes du corpus révèle des variations importantes. Le lecteur entame alors un travail de genèse en comparant les manuscrits comme autant de variations sur un thème qui se cristallise de plus en plus comme biographème. A l'instar de la critique génétique qui interprète les suppressions, les adjonctions, les déplacements et les permutations dans les différents états du manuscrit, le lecteur apaïste reconstruit la genèse du biographème à partir de ces mêmes opérations qui sont à l'origine des réécritures successives d'une même configuration. Dès lors, l'ensemble du corpus forme texte et les variations peuvent être interprétées comme des lieux de non-dit dont il faut rechercher la signification. L'enfant, élevé au village par sa grand-mère, ignore la raison pour laquelle ses parents l'ont confié à ses grands-parents paternels. Il ne retrouve ses parents à la ville que pendant les vacances. Un non-dit pesant entoure cette séparation. Dans l'implicite, les mœurs de ses parents sont condamnées: la relation triangulaire laisse sous-entendre une relation homosexuelle du père avec son ordonnance que l'enfant de neuf ans ne peut comprendre. Les parents sont absents au moment de l'exode. On en cache la raison à l'enfant. Lors du retour, la grand-mère qui a différé la révélation, annonce à l'enfant leur décès dans un bombardement.

L'enfant apprendra plus tard qu'ils sont effectivement morts en 1938, donc avant la guerre, dans un suicide au cap Blanc Nez en France, l'ordonnance ayant disparu. Dans ce scénario, le grand-père du personnage est tué pour délit sexuel, pendant l'exode. Le déplacement principal concerne l'homosexualité. Dans les autres versions ce n'est pas le père mais le grand-oncle (le frère de la grand-mère) à qui est attribué ce biographème de la liaison homosexuelle avec l'ordonnance ${ }^{5}$. Le triangle classique est composé de la mère, du père de la maîtresse et le grand-

\footnotetext{
${ }^{5}$ Mémoire d'une jeune vie dérangée, p. 72.
} 
père et les parents ne disparaissent pas pendant la guerre. Le travail d'interprétation du lecteur apaïste ne vise pas à chercher la vérité (la vraie biographie) mais cherche à comprendre le sens du non-dit de ce déplacement. A partir de cette interrogation centrale, une construction de lecture se met en place, cohérente et féconde. De tels déplacements d'items dans le biographème sont lourds de significations non explicites. La manipulation d'écriture qui consiste à attribuer l'homosexualité du grand-oncle au père contribue à augmenter la tension de la problématique du noyau familial. Cette condensation opérée dans la constellation familiale par la mise en discours pour révéler le non-dit déploie toute son efficacité à travers le cheminement du mot «ordonnance». L'enfant perçoit d'abord le signifiant du signe linguistique: on parle autour de lui de l'ordonnance qui l'empêche d'être élevé par ses parents. Il accède ensuite au référent, à la chose désignée par le mot, en voyant pendant l'exode «une grosse ponte avec son ordonnance», une ordonnance en chair et en os et l'enfant de s'écrier: «Ah, c'est cela une ordonnance!! ${ }^{6}$. Plus tard, la signification de cette fonction sera recherchée et conduira à l'accès du signifié du mot. Cet accès au non-dit (l'homosexualité) par ce trajet de l'accès au signe linguistique pour découvrir un mot et le comprendre est comme un ordonnancement du sens, du vécu, réplique des sens «d'ordre», «d'ordonner les choses» que ce mot évoque. La manière dont ce mot chemine dans le vécu de l'enfant trahit qu'il s'agit pour le je de parler de trauma. Ces indices et la page 140 par sa haute condensation scripturale, au sens où un rêve ou un conte condense une énigme en en projetant la tension dans une image forte, seront placés au centre de la construction interprétative du travail de lecture comme un noyau dur:

Lundi 20 mai. Les mots fustigeant les rapports de mes parents avec l'ancienne ordonnance résonnaient toujours à mon oreille, bien que leur réalité crue fût moins, en ce qui me concernait, un problème d'acceptation (la question ne se posait pas de cette façon à mon âge), qu'une interrogation lancinante sur la suspicion larvée, la désapprobation muette, l'opprobre permanent, qui stigmatisaient le lien formé entre ces trois personnages, et qui les embastillaient au plus secret d'une forteresse inaccessible, bardée de limbes glacières, pétrifiées par un silence et une absence irrémédiables ${ }^{7}$.

Cette image hyperbolique, quasi féerique du retrait de toute vie de ce monde froid, blanc et rigide, place l'enfant hors du monde comme si le secret qu'il doit partager malgré lui avec ses parents, en le faisant participer à l'innommable, l'empêchait, lui, de partager avec le monde

${ }^{6}$ Un exode en mai, p. 59.

${ }^{7}$ Un exode en mai, p. 140. 
extérieur, isolé, emmuré par la terreur et le froid du triangle. Pour l'enfant, la filiation est inavouable, le secret de glace le rejette dans l'inexistence car il n'est pas accompagné par les trois protagonistes du triangle, trop occupés d'eux-mêmes. D'autant plus que la mère semble complice et subit la situation «malsaine» qui n'arrête pas d'exister. Comment dès lors, se faire une image de soi pour se relier à la société et se construire ou sortir de soi? La gravité de cette interprétation à laquelle est amené le lecteur qui interprète ainsi un difficile accès à la résilience est bien une lecture du non-dit puisque c'est la construction de la genèse des manuscrits qui y invite en plaçant dans l'abyme, au cœur de l'œuvre, ce texte le plus éloigné du scénario leitmotiv.

Informée, par cette construction lectorielle, de l'importance du contenu sous-jacent - la béance créée par le non accompagnement que l'écriture entreprend de combler -, la cohérence de la disposition en symétrie des autres textes du corpus autour de celui-ci apparait limpide. S'il a fallu faire l'expérience du réfugié pour réaliser l'absence d'accompagnement parental en étant jeté hors du monde, le récit de ce biographème déborde le drame personnel pour instaurer l'écriture comme un outil de connaissance de soi, du monde, afin de sortir du palais des glaces létales. A la suite de cette interprétation de l'exode comme la mise à jour, à travers un drame personnel, de certains impensés sociétaux en vue de leur élaboration, encore peu envisagée à l'heure actuelle en tant que traumatismes subis par les jeunes enfants de la guerre de 1940, les autres textes du corpus peuvent être lus à la lumière de la même thèse implicite.

Un retour au village et sa réécriture, Mémoires d'une jeune vie dérangée mettent à jour la raison, en quelque sorte futile du secret révélé après coup, ce qui n'enlève rien à la quête «archéologique» du sens que l'écriture entreprend, ni à l'ampleur du trauma qui la commande. Cette insignifiance d'apparence - la jalousie du père - n'est donc pas la raison véritable de la quête mais l'inscription de cette quête dans un contexte politique, celui de la guerre, celui de l'émigration des campagnes et de la mutation de la société quittant les repères du patriarcat rural.

Avatar à Baranda et sa réécriture, Les jouets cassés de la Congolie, translatent la même thèse d'un impensé sociétal non élaboré, - le colonialisme vécu par de jeunes adultes arrivés au moment où l'identification à la filiation triomphaliste n'était plus possible. Cinquante an après, cet impensé n'est pas encore élaboré comme traumatisme social. Entre ces deux épisodes autobiographiques qui concernent le Congo belge, c'est également la mise à jour d'un non-dit primordial que l'écriture se propose de rendre dicible. Là aussi, les déplacements d'items du biographème jouent leur rôle comme dans les rêves, la seconde version révélant les condensations de la première. Par exemple le fait que le contrat dans la colonie se répartisse sur deux termes de trois ans dans Baranda, alors qu'il n'est pas renouvelé dans La Congolie, permet 
d'attribuer au personnage une liaison avec une ménagère dont il a eu un enfant avant son mariage, concentrant ainsi en une seule intrigue les dilemmes conjugaux. D'autres modifications ténues d'une scène récurrente du corpus indiquent la forte implication affective du segment du scénario autobiographique concerné. Une telle scène va jusqu'à être réécrite trois fois. Dans les deux textes concernant le Congo cette scène décrit le sacrifice de la chèvre qui est une «reconstitution d'une exécution, telle qu'elle se pratiquait dans les guerres tribales, lorsque les Ngombés sacrifiaient leurs prisonniers» ${ }^{8}$ : «Les hommes serraient les mains de leurs épouses, de leurs filles, froides au creux des leurs» ${ }^{9}$; «Bertrand serra la main de sa femme, toute froide au creux de la sienne» ${ }^{10}$. Dans l'Exode, une configuration identique dans la construction du point de vue autorise sa superposition aux deux précédentes même si le spectacle observé ne traite pas du même sujet. L'existence d'une version «originelle» de ce motif dans le texte central fournit un exemple complet de la genèse de l'écriture de ce corpus. Il s'agit de la scène de l'abattoir improvisé pendant d'exode et observée par Céline et le narrateur enfants: «Un rictus se lisait sur le visage des curieux que le carnage fascinait. La main de Céline s'agrippait à la mienne. Elle sursautait à chaque coup de masse qui s'abattait sur le crâne des bovidés, et à la seconde précise où le sang jaillissait sous la lame de l'égorgeur» ${ }^{11}$.

\section{La configuration du non-dit dans le discours autobiographique de José Dosogne}

Le non-dit traverse donc le texte à deux niveaux:

1. Il est le lieu pour le scripteur de la principale commande d'écriture: écrire pour transformer l'informulé traumatisant en significations dicibles, pas uniquement au niveau du roman familial personnel, mais aussi au niveau politique au sens large comme un devoir individuel d'advenir en tant que protagoniste impliqué dans le monde. Ce non-dit se lit au niveau de l'explicite et à ce titre, chaque volume est un roman à thèse dont les incipits énoncent les intentions. Par exemple pour le texte que j'ai placé au centre de la configuration: «Les exodes de populations demeurent par excellence l'illustration métaphorique des outrances

\footnotetext{
${ }^{8}$ Congolie, p. 70 .

${ }^{9}$ Idem.

${ }^{10}$ Baranda, p. 143.

${ }^{11}$ Un exode en mai, pp. 104-105.
} 
F. Meurice: Le travail de lecture des groupes de lecteurs de l'APA-Bel

de la guerre et des tribulations de la destinée humaine» est la thèse qui est placée sur le seuil du récit de l'exode entamé comme un voyage de révélation.

2. Il est le lieu pour le lecteur de la principale commande de coopération dans la lecture, de la construction de l'interprétation. Ce sont les variations avec déplacements, permutations, adjonctions et suppressions d'items dans le scénario leitmotiv mais aussi dans les métaphores obsédantes et récurrentes, les superpositions de scénographies, les répétitions des mêmes prénoms, les tressages des couches énonciatives qui alimentent la lecture de l'implicite. La narration événementielle est soit accompagnée d'une sous narration contextuelle, construite a posteriori et destinée à renforcer la reliance de l'événement relaté au contexte sociopolitique contemporain, soit superpose les couches temporelles comme dans Un retour au village entremêlant l'énonciation de l'enfance et celle du scripteur autobiographique. Dans ces deux cas de figure, cette manière de raconter approfondit le travail que la commande d'écriture déchiffrée permet de supputer: sortir de la mise hors du monde en se forgeant soi-même ses liens au monde. Ce registre d'interprétation du non-dit est de l'ordre de l'implicite et est donc une construction lectorielle.

\section{Conclusion. La lecture apaïste}

Notre exploration de la posture lectorielle d'un corpus de textes du patrimoine archivé des autobiographies a redessiné les mêmes relations possibles entre un texte, son auteur et son lecteur que celles qui furent chronologiquement vécues par l'histoire de l'enseignement de la littérature. Chercher la révélation patrimoniale d'un texte en le lisant à travers la biographie de son auteur, c'est privilégier la figure de l'auteur comme le faisait l'école de Gustave Lanson dans les années 1970 et se heurter dans le cas des autobiographies à la question stérile de la vérité factuelle. Considérer que la lecture littéraire c'est uniquement la lecture des textes littéraires, c'est faire fi de la participation du lecteur qui coopère à l'émergence du sens, et des théories de la réception qui ont vu le jour dans les années 1990, c'est s'écarter également de la mission d'éducation populaire inhérente à l'APA. Lire le texte pour lui-même en le considérant comme un espace se suffisant à lui-même, c'est se conformer à la représentation de la lecture littéraire des années 1980 illustrée par les grilles structurales de la narratologie. Se contenter d'une lecture en sympathie suppose une lecture littéraire participative et rejoint la représentation de la lecture plaisir hostile à l'analyse. La dernière approche, qui fut la nôtre, est celle qui cumule distanciation et participation. Associer une distanciation à la posture de la lecture 
hédoniste permet au lecteur d'entrer dans le jeu du plaisir de lire mais le rend aussi capable d'analyser la partie jouée ${ }^{12}$. La didactique de la lecture littéraire revendique actuellement cette représentation. Notre cheminement nous conduit à préférer le terme d'empathie pour caractériser la lecture de l'échotier apaïste au détriment de l'expression lecture en sympathie - l'empathie maintenant cette distance capable d'analyse ${ }^{13}$.

Mettre l'accent sur les compétences du lecteur apaïste dans l'élaboration de l'interprétation d'un dépôt permet d'identifier trois lieux de tension inhérents au dispositif communicationnel de l'APA:

1. Le lecteur oscille entre une attitude participative et une attitude distante. L'attitude distante le conduit à envisager l'écho plutôt en empathie qu'en sympathie et à analyser ses propres constructions interprétatives du non-dit comme une genèse de l'écrit autobiographique.

2. Le lecteur opère une réception imaginaire des textes déposés à l'APA sur le mode autographique $^{14}$, les manuscrits sont envisagés comme des œuvres se manifestant par ellesmêmes à l'instar d'un tableau, d'une sculpture, d'une performance alors que se sont des textes de type allographique ${ }^{15}$ comme une partition, un livre, un scénario qui demandent à être mis en œuvre par le lecteur. C'est la mise en scène communicationnelle de l'APA qui n'accueille que des manuscrits non édités et met en présence physique les deux pôles de la production (auteur et déposant) et de la réception (lecteur et scripteur de l'écho) qui induit ce régime d'existence du texte, objet unique du patrimoine.

3. Le lecteur est amené à explorer d'autres authentifications auctoriales lorsque l'écriture se manifeste en versions successives. Dans ce cas, l'œuvre déborde son immanence, c'est ce que nous avons indiqué en textualisant le corpus, en le traitant dans l'intertextualité. Le pacte de lecture de l'autobiographie est déstabilisé dans ce cas de figure des œuvres à versions successives, lacunaires (fragments) ou indirectes (copie, reproduction, description $)^{16}$.

\footnotetext{
${ }^{12}$ M. PICARD, «La lecture comme jeu», 1986, in D. DELBRASSINE, Découvrir 'la lecture littéraire' avec les romans écrits pour la jeunesse, op. cit.

${ }^{13}$ Cf. la distinction de Carl ROGERS, «A Theory of Therapy», in A. LAINE, op. cit., p.49.

${ }_{15}^{14}$ Gérard GENETTE, L'œuvre de l'art, Immanence et transcendance, Paris, Seuil, Poétique, 1994.

${ }^{15}$ Idem.

${ }^{16}$ Cf. classification des régimes d'existence des œuvres d'art GENETTE (ibidem).
} 
F. Meurice: Le travail de lecture des groupes de lecteurs de l'APA-Bel

Au terme de ce parcours, le lecteur apaïste se profile comme un actant de forces lectrices agissantes en ces trois lieux de tension. 